International Journal of Transdisciplinary AI

Vol. 3, No. 1 (2021) 25-41

(C) KS Press, Institute for Semantic Computing Foundation

DOI: $10.35708 /$ TAI1870-126251

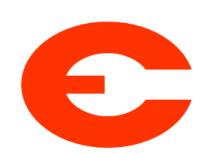

\title{
Estimating Number and Dwell Time of Visitors in a Large-scale Indoor Space from HVAC Data and Its Evaluation in Different Seasons
}

\author{
Keisuke Tsunoda, Naoki Arai, and Kazuaki Obana \\ NTT Smart Data Science Center, Nippon Telegraph and Telephone Corporation, \\ 1-8-1, Shibaura, Minato, Tokyo Japan \\ \{keisuke.tsunoda.cx, naoki.arai.zd, kazuaki.obana,ax\}@hco.ntt.co.jp
}

Received $(12 / 15 / 2020)$

Revised $(02 / 01 / 2021)$

Accepted (02/15/2021)

\begin{abstract}
The aim of this paper is to estimate the number and dwell time of visitors in a large-scale indoor space or room with a common heating-ventilation-air conditioning (HVAC) system that includes sensors for $\mathrm{CO} 2$ and indoor temperature in any season. Previous studies tried to estimate the number and dwell time of visitors from $\mathrm{CO} 2$ concentration in small rooms with or without a HVAC system. However, in a large-scale indoor space with large air-conditioning and ventilation systems, the number and dwell time of visitors are difficult to estimate for three reasons: 1) CO2 concentration changes much more slowly than the number and dwell time of visitors and with a delay, 2) the difference in changes is affected by the amount of ventilation, and 3) this difference may be affected by operation of HVAC, which is affected by seasonal climate. To solve these problems, we proposed partial modeling with a variable time window. This method can make a partial estimation model that automatically corresponds to differences in the change speed between two variables: visitors and $\mathrm{CO} 2$ concentration. We demonstrate the effectiveness of our proposal using measured sensor data in summer, fall, and winter to clarify its feasibility in different seasons in Japan.
\end{abstract}

Keywords: People flow; Partial modeling with Variable Time Window; HVAC data, Seasonal effect, Facility management

An earlier version of the paper was presented at the Third International Conference onTransdisciplinary AI. 


\section{Introduction}

Large urban facilities such as shopping complexes, train stations, airport buildings, and office buildings are not only spaces for shopping, business, or transportation but also important infrastructure for city residents. To enhance their value, facility managers and operators need to operate and maintain them efficiently and effectively with digital technology [1. To properly manage, maintain, and operate such facilities, one of the most important data is people flow data including the number of visitors and their dwell time during each time period and day because these data suggest the use pattern of the facility, best schedule for air-conditioning [2], and best time or day for maintenance and construction [3. People flow data are generally measured by multiple sensors such as cameras [4] or laser range finders (LRFs) [5]. However, these are expensive to install, maintain, and operate because many sensors and a complex system for aggregating or analyzing data from these sensors are necessary to measure people flow in a large room or space. To solve this problem, previous studies tried to estimate people flow, especially the number of visitors or number $\times$ dwell time of visitors by using $\mathrm{CO} 2$ concentration [6, which is affected by visitors in a room. However, people flow in large space or room is difficult to estimate using $\mathrm{CO} 2$ concentration for three reasons: 1) $\mathrm{CO} 2$ concentration changes much more slowly than the number and dwell time of visitors and with a delay, 2) this difference in changes is affected by the amount of ventilation, and 3) this difference may be affected by operation of heating-ventilation-air conditioning (HVAC), which is affected by seasonal climate.

To solve these problems, this paper introduces our proposed method, partial modeling with a variable time window, which can be used to estimate people flow including the number and dwell time of visitors by using existing $\mathrm{CO} 2$ concentration as a part of HVAC system, a building automation system (BAS) [7] or building energy management system (BEMS) 8$]$ system in a large-scale indoor space such as a lounge or hall. The effectiveness of our proposal is evaluated using data from three different seasons to clarify its seasonal effect in Japan.

\section{Related works}

\subsection{Direct sensing of people flow}

There are various solutions to measure people flow. For example, security cameras can be used, although they may present a privacy problem. To solve this privacy problem, multiple electro-optical devices such as LRF [5] and light detection and ranging (LiDAR) 9] can be applied to measure people flow in a large room or space with many people. These solutions can measure people flow more accurately, but to use them in a large space or room, multiple LRFs must be installed. In addition, multiple sensors require a complex network environment and data aggregating and analyzing system such as people detection and tracking. Therefore, multi-sensor solutions cost more to not only install but also operate and maintain than single-sensor solutions, especially in a large space or room with a lot of people. 


\subsection{Indirect sensing and estimation of people flow}

To solve the privacy problem, indirect methods to estimate people flow have been proposed. For example, Longo et al. proposed a method to estimate the number of people using Bluetooth or Wi-Fi signals emitted by visitors' devices [10. Mateos-Sánchez et al. tried to estimate occpancy in indoor and outdoor space using BLE and open data [11. However, these methods cannot sense or estimate people without devices that emit such signals. Zou et al. proposed a method to estimate the number of people using propagation and reflection of WiFi signals in a smart building without visitors' devices [12. However, their proposal has been applied to estimate fewer than 12 people in a room about $8 \mathrm{~m} \times 8 \mathrm{~m}$. Therefore, their proposal seems difficult to use to estimate people flow in a large space or room with many more people.

Arief-Ang et al. tried to estimate the number of people in a small room and large hall by using a $\mathrm{CO} 2$ concentration sensor [6]. In a large facility, a method using $\mathrm{CO} 2$ concentration is cheaper to install, maintain, and operate than direct sensing methods because $\mathrm{CO} 2$ concentration sensors are already installed and used in smart buildings with HVAC, BEMS or BAS. However, in a large facility where a lot of people go in/out frequently, the number of people is difficult to estimate with a general estimation model with a fixed time window because the facility also has air-conditioning or ventilation systems, which affect the difference in change speed between the number and dwell time of visitors and $\mathrm{CO} 2$ concentration.

\subsection{Indirect estimation with HVAC system}

In HVAC system, not only temperature and humidity but also the volume of air supply and $\mathrm{CO} 2$ concentration, which is useful for estimating the number and dwell time of visitors, are measured for controlling the environment in a room. Therefore, studies have tried to estimate the number of visitors in a room using HVAC system data including CO2 concentration. For example, Ebadat et al. tried to estimate the occupancy level, which was at most five people, in a small room using standard HVAC system data and a fused-lasso regression model [13]. Alam et al. tried to estimate the occupancy level, which was at most five people, in a small room with automated ventilation system using a dynamic neural network with the number of neurons and tapped delay lines [14. However, previous studies have only tried to estimate at most several people in a small room such as a class room or small office.

\section{Requirements and Issues}

\subsection{Requirements}

In this section, we try to extract requirements to estimate people flow in a large space or room in a large facility.

First, we focus on requirements for people flow estimation on the basis of the needs of facility managers and operators. In general, one of their most important tasks is maintenance, including cleaning, air-conditioning, and repairing 
facilities. Specifically, they want to clean and repair facilities during times and days with few people and without dynamic people flow. For air-conditioning, they want to maintain a comfortable air temperature and humidity and save energy in response to the number and dwell time of visitors in rooms because human body temperature affects room temperature. Another important task is to promote their facility to tenants and visitors. To enhance the value of their facility, they must determine how many people come into the facility for how long because they can analyze and understand the use pattern of the facility on the basis of such data. Therefore, facility managers and operators need to estimate people flow data including the number and dwell time of visitors.

On the other hand, facility managers and operators also need to consider the costs of sensing and estimation, i.e., the costs of installing, maintaining, and operating the sensors and system. Especially, to estimate people flow in a large room or space, a solution is required that has many sensors and a complex data aggregation and analysis system. Therefore, people flow needs to be estimated without many sensors or a complex system but with existing or few sensors.

In addition, a large space or room in such facilities can contain many people, and the number of people can dynamically change. Therefore, a method is needed that can estimate many visitors, e.g., more than 500, who go frequently in/out of a large-scal indoor space such as a lobby or hall.

Lastly, since HVAC operation is changed to control temperature and humidity during all seasons, facility management using people flow is needed at all times in all seasons. Therefore, a method is needed that can estimate the number and dwell time of visitors in all seasons.

As a result, four requirements are extracted.

1 Estimating the number and dwell time of visitors in a large room or space.

2 Using only existing sensors in a facility, such as sensors in a HVAC or BEMS.

3 Estimating large numbers of people (more than 500) who go in/out of a large space or room.

4 Estimating the number and dwell time of visitors in any season.

\subsection{Problem}

In this section, we clarify the main problem in this paper on the basis of the requirements described above. In general, large facilities have a BEMS or BAS that includes $\mathrm{CO} 2$ concentration sensors. Estimating people flow on the basis of these systems and sensors is much cheaper than other approaches with multiple LRFs, cameras, and so on. However, in such facilities, people flow is difficult to estimate from $\mathrm{CO} 2$ concentration because not only does $\mathrm{CO} 2$ concentration change much more slowly than the number and dwell time of visitors and with a delay but also these differences change depending on the amount of ventilation in HVAC operation, which is affected by seasonal climate. In particular, these difficulties seem to be more critical in large-scale indoor spaces.

Therefore, the main problem to overcome is to estimate the number and dwell time of visitors in a large space or room using CO2 concentration data measured from HVAC or BEMS in response to differences in their change speeds 
and changes in these differences caused by air-conditioning or ventilation, which is affected by seasonal changes in temperature and humidity.

\section{Method}

To solve this problem and satisfy requirements 1-3, we proposed a novel method (partial modeling with a variable time window) and evaluate it with limited data in specific seasons [15. In this paper, we evaluate our proposal with three different seasonal datasets to clarify its feasibility in different seasons and whether it satisfies all four requirements.

Here, we introduce details of our proposed method: partial modeling with a variable time window. Partial modeling is a method to learn and make a model with selected learning data similar to a target point [16] and has been applied to make the model with non-linear and complex systems such as a chemical plant [17. We extend it to apply it to variable changes in relationships between $\mathrm{CO} 2$ concentration and the number and dwell time of visitors by selecting a time window in the model. Fig. 1 shows the flow of our proposal to estimate the number and number $\times$ dwell time $(\mathrm{sec})$ of visitors. Our proposal consists of two phases: 1) make models with multiple patterns of the time window $\delta t$ and number of selected learning data $n$, and 2) select the model with the most suitable $\delta t$ and $n$ and use it to estimate the number of visitors or number $\times$ dwell time of visitors.

In phase 1 , first, initialize the parameters: target time $t$ for the estimation, maximum of time window $\delta t_{\max }$, minimum of time window $\delta t_{\min }$, maximum number of data $n_{\max }$, and minimum number of data $n_{\min }$ for modeling. Initialize variables $\delta t=\delta t_{\text {min }}, n=n_{\min }$. Then, obtain explanatory variables: CO2 concentration (CO2), inverter output of air supply (INV output), and difference in $\mathrm{CO} 2$ concentration from a recent time window (CO2 dif) from time $t-\delta t$ to $t+\delta t$ and combine them as vector $X$. Next, for each timestamp $t^{\prime}$ in the database (DB), extract variables CO2, INV output, CO2dif, and target variable; number of visitors or number times dwell time of visitors from $t^{\prime}-\delta t$ to $t^{\prime}+\delta t$ and make vector $X_{a l l}$ and $Y_{a l l}$, respectively. To select learning data, calculate dist $t_{t . t^{\prime}}$ on the basis of formula (1). Then, select $X_{t^{\prime}}$ and $Y_{t^{\prime}}$ on the basis of dist $t_{t t^{\prime}}$ and $\mathrm{K}$-nearest neighbor $(\mathrm{k}-\mathrm{NN})$ method with $n$ and learning data vector $X_{\text {learn }}$ and $Y_{\text {learn. }}$.

$$
\text { dist }_{t . t^{\prime}}=\left|X_{t}-X_{t^{\prime}}\right|
$$

Therefore, make model $\alpha$ on the basis of formula (2) and calculate the model's

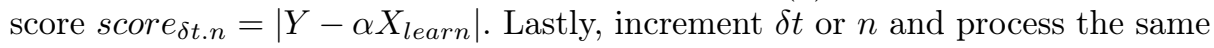
flow until $\delta t_{m} a x$ or $n_{m} a x$, respectively.

$$
Y_{\text {learn }} \approx \alpha \times X_{\text {learn }}
$$

In phase 2 , extract the best model $\alpha^{*}$, time window $\delta_{t}^{*}$ on the basis of the minimum score. Then, extract explanatory variables; CO2, INV output, and CO2 dif from $t-\delta t^{*}$ to $t+\delta t^{*}$ as vector $X$. Lastly, estimate the number of visitors or number times dwell time of visitors $\hat{Y}_{t}$ on the basis of formula (3). 


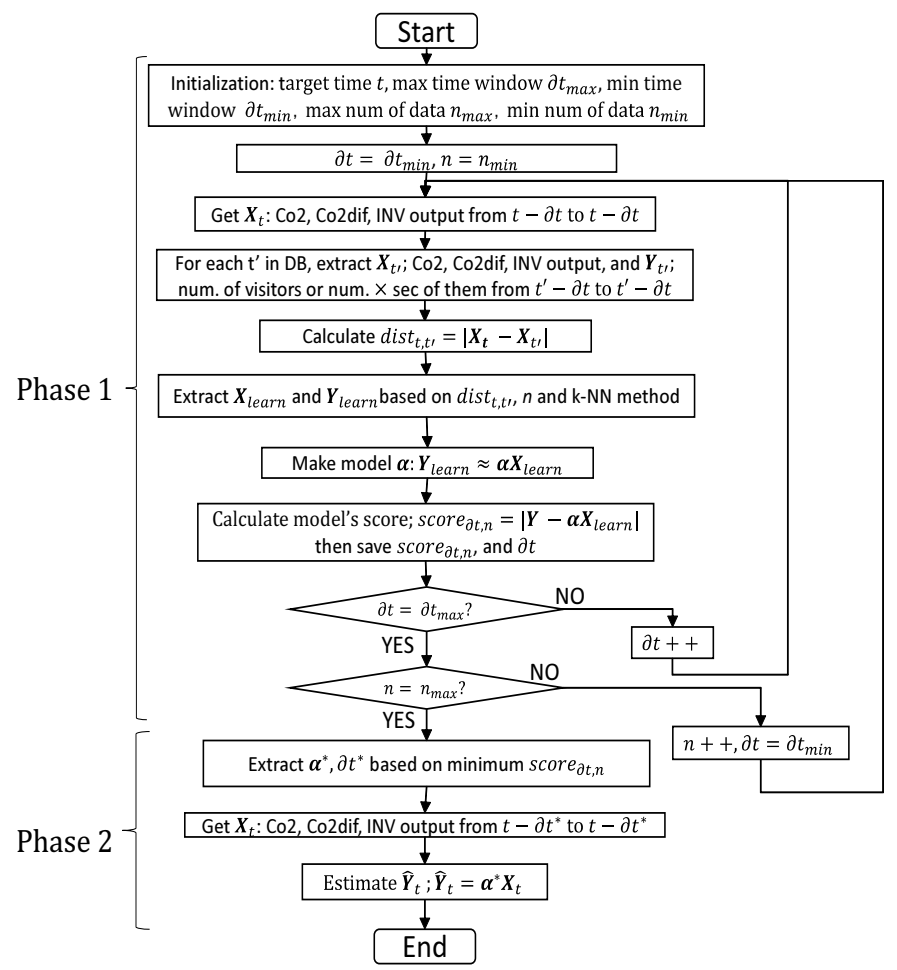

Fig. 1. Flow of our proposed method

$$
\hat{Y}_{t}=\alpha^{*} \times X
$$

\section{Evaluation}

\subsection{Target facility and space}

We have evaluated our proposal with data measured in the lobby of a building containing both shops and offices. Fig. 2 shows the layout of the target lobby, which is about 25 meters $\times 10$ meters $\times 10$ meters (height). For measuring correct data, seven LRFs were installed that measured the number of visitors, which is defined as the number of unique people in the lobby at each $10 \mathrm{~min}$, and the number $\times$ dwell time of visitors, which is defined as the summation of dwell time of each visitor in the lobby at each $10 \mathrm{~min}$.

\subsection{Evaluation settings}

Data continuously collected over 198 days (from Aug. 1. 2019 to Feb. 15. 2020) were used for the evaluation. In our previous research, only 14 days of fall data were used as test data to evaluate the basic effectiveness of our proposal. In this paper, to evaluate the effectiveness of our proposal in summer, fall, and winter, data measured on 14 selected days in each season (summer: from Aug. 19. to 


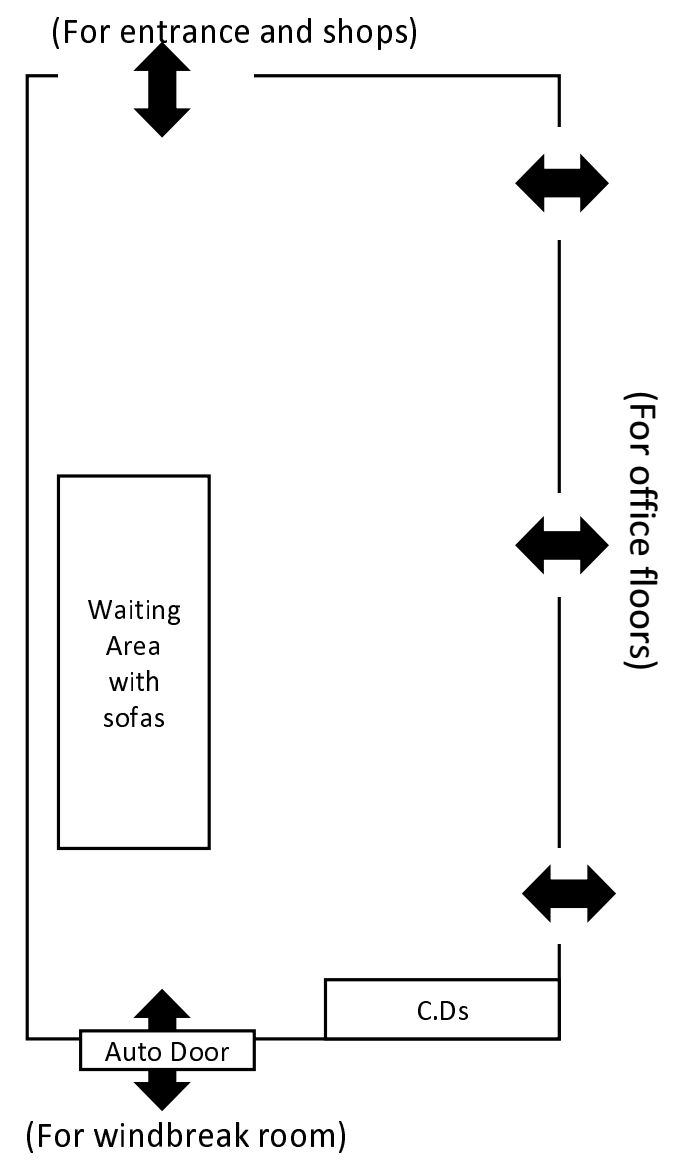

Fig. 2. Target lobby

Sep. 1. , fall; from Oct. 15. to Oct. 28. and winter; from Jan. 11. to Jan. 24, 2020) were used as test data, and the remaining data were used as learning data. We choosed these seasons because in Japan, temperature in spring is similart to that in fall [18. The features in the evaluation are CO2 concentration (CO2), inverter output of air supply (INV output), and difference in CO2 concentration from recent time window (CO2 dif). Target variables are the number of visitors and number $\times$ dwell time of visitors, which were evaluated separately. Their time window is $10 \mathrm{~min}$.

Our proposed time window is variable from 3 (30 min.) to 12 (120 min.), and the number of selected learning data is also variable: 25, 50, 75, and 100 . Support Vector Regression (SVR) [19] was used for the estimation.

For comparison, basic SVR models with a predefined time window (from 3 to 12 ) were applied to the same estimation. 


\subsection{Results}

Fig. 3 shows results for the correlation coefficient $r$ in estimating the number of visitors by each method for each season. Fig. 4 shows results of the ratio of mean squared error (RMSE) in estimating the number of visitors by each method for each season. Fig. 5 shows results for the mean squared error (MSE) and its paired t-test in estimating the number of visitors by each method for each season. The results show that our proposal can estimate the number of visitors more accurately than all comparison methods significantly.

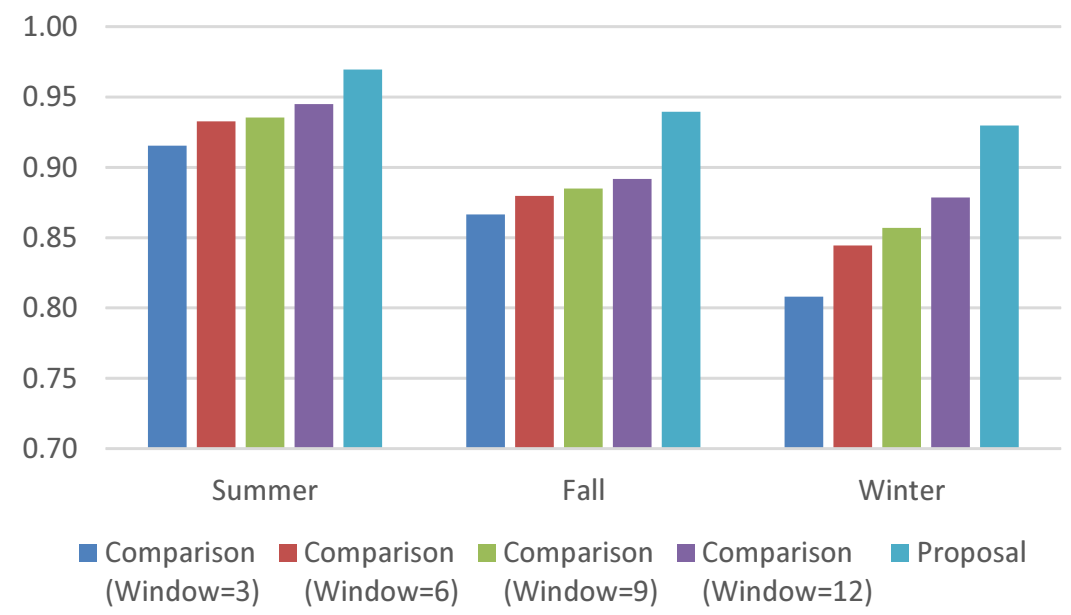

Fig. 3. Results for the correlation coefficient $r$ in estimating the number of visitors by each method for each season 


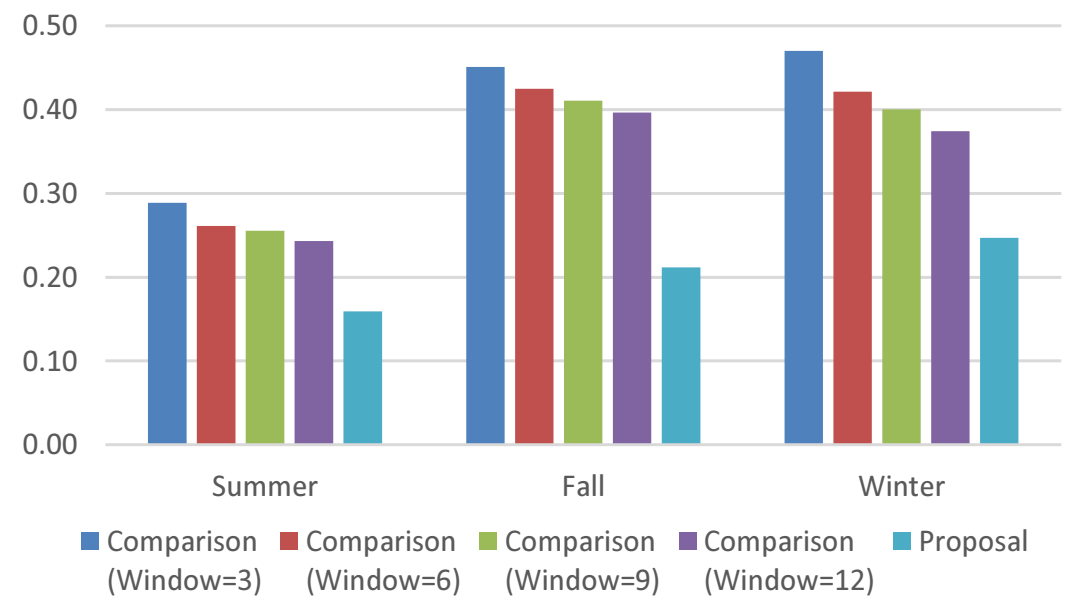

Fig. 4. Results for the ratio of mean squared error (RMSE) in estimating the number of visitors by each method for each season

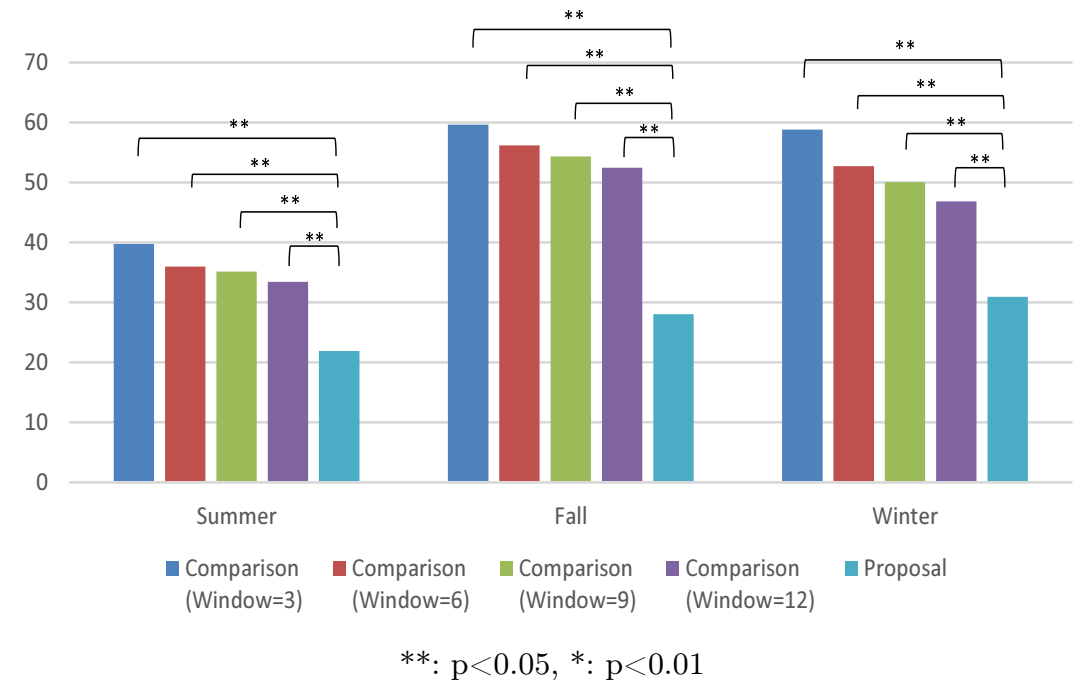

Fig. 5. Results for the mean squared error (MSE) and its paired t-test in estimating the number of visitors by each method for each season

Fig. 6. 7. and 8 show an example of the results and length of time window selected by our proposal in estimating the number of visitors on a certain weekday in summer, fall, and winter, respectively. 


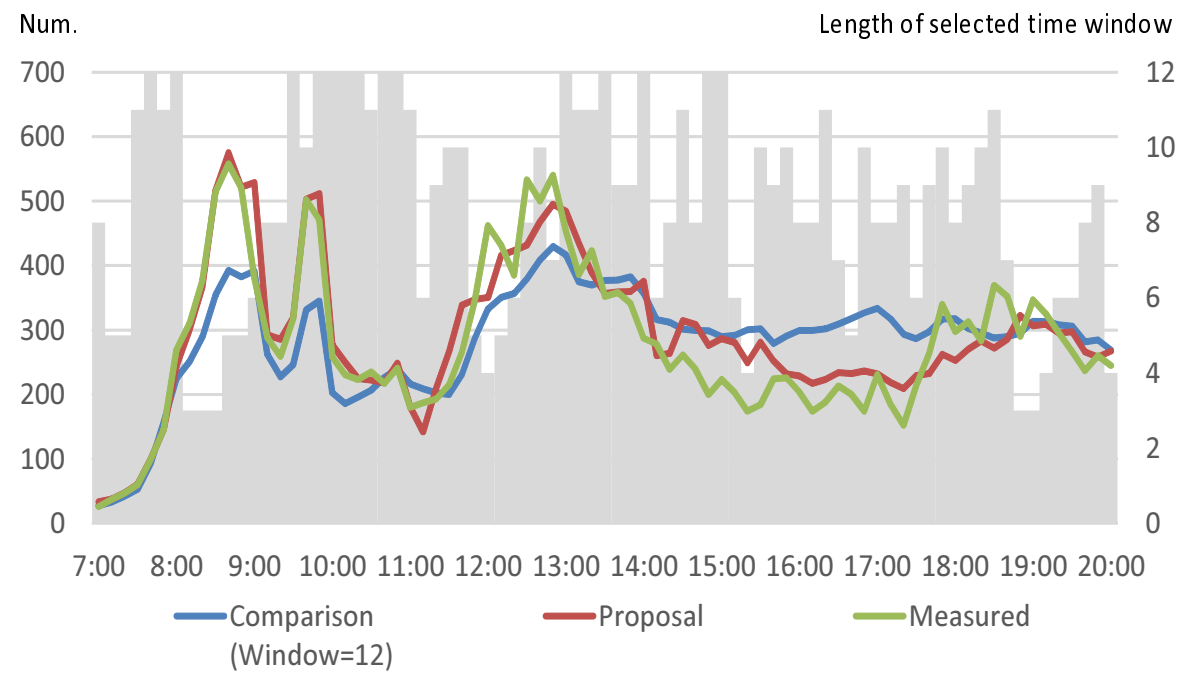

Fig. 6. Results of each method for estimating the number of visitors and length of time window selected by our proposal on a certain weekday in summer

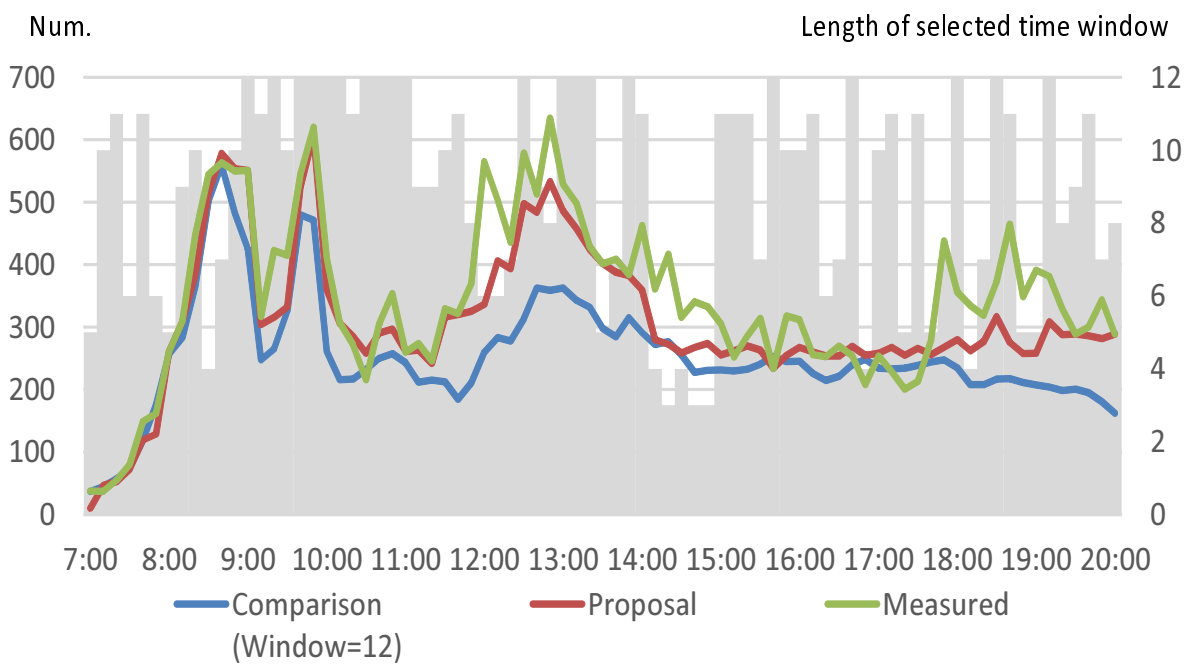

Fig. 7. Results of each method for estimating the number of visitors and length of time window selected by our proposal on a certain weekday in fall 


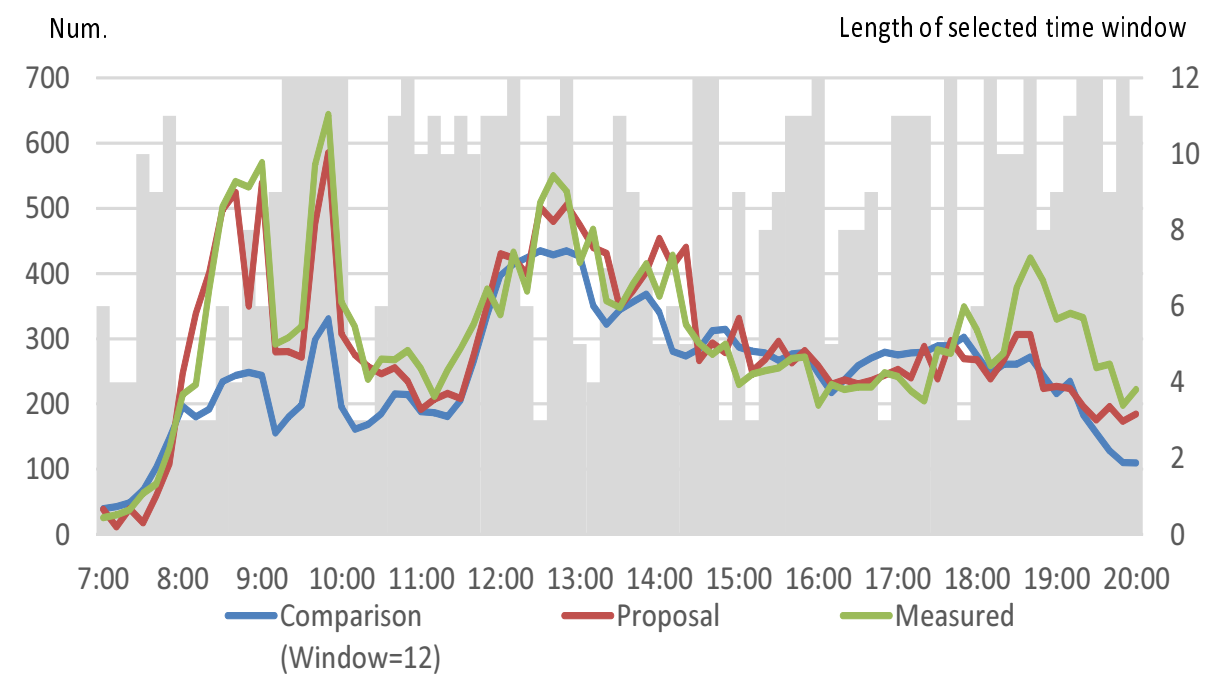

Fig. 8. Results of each method for estimating the number of visitors and length of time window selected by our proposal on a certain weekday in winter

Fig. 9 shows results for the correlation coefficient $r$ in estimating the number $x$ dwell time of visitors by each method for each season. Fig. 10 shows results of the RMSE in estimating the number $\times$ dwell time of visitors by each method for each season. Fig. 11 shows results for the MSE and its paired t-test in estimating the number $x$ dwell time of visitors by each method for each season. The results show that our proposal can estimate number $\times$ dwell time of visitors more accurately than all comparison methods significantly. 
1

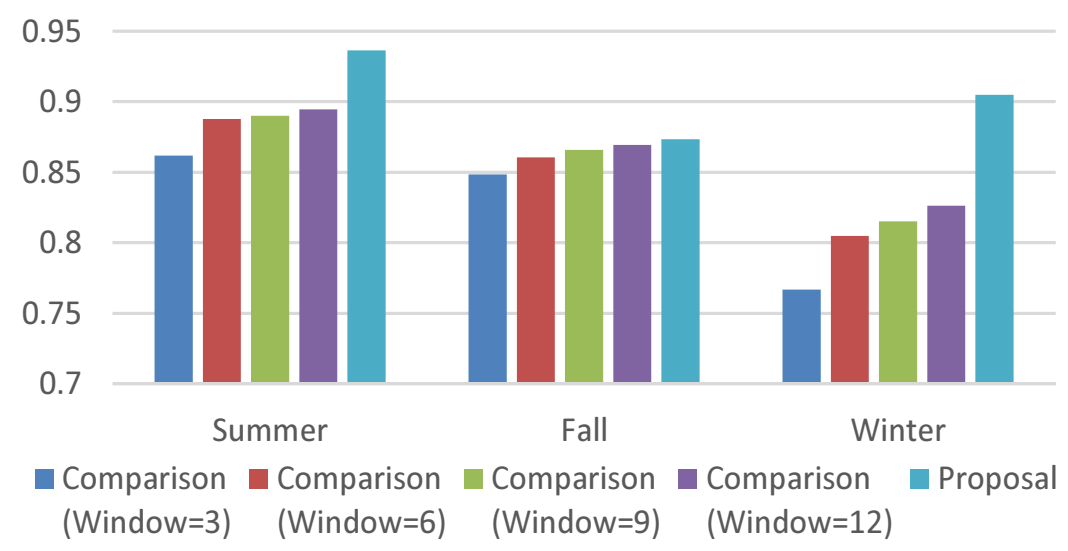

Fig. 9. Results for the correlation coefficient $r$ in estimating the number $\times$ dwell time of visitors by each method for each season

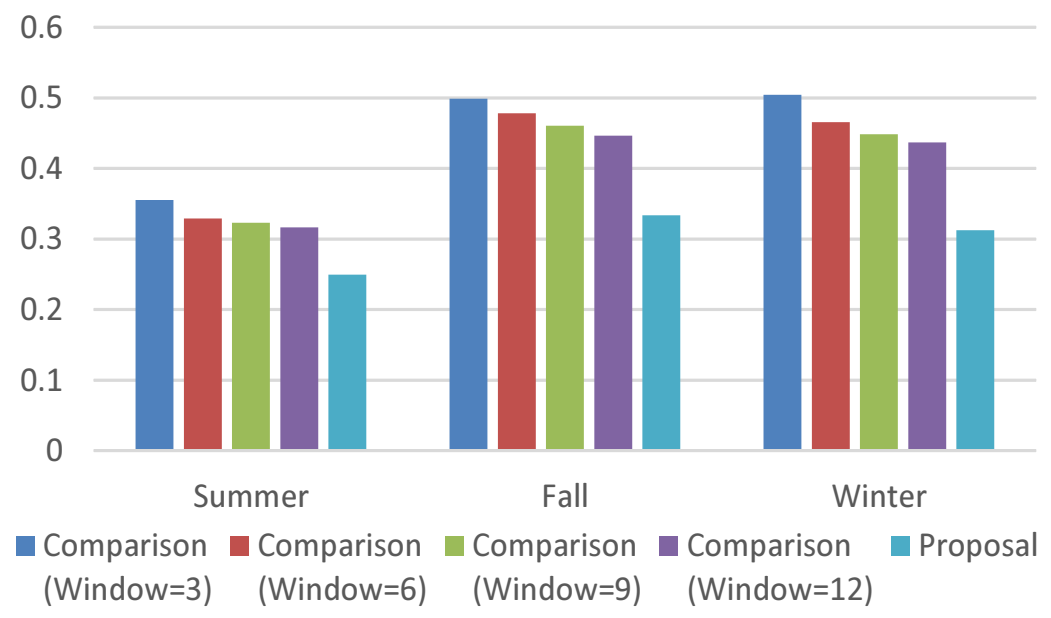

Fig. 10. Results for the RMSE in estimating the number $\times$ dwell time of visitors by each method for each season 


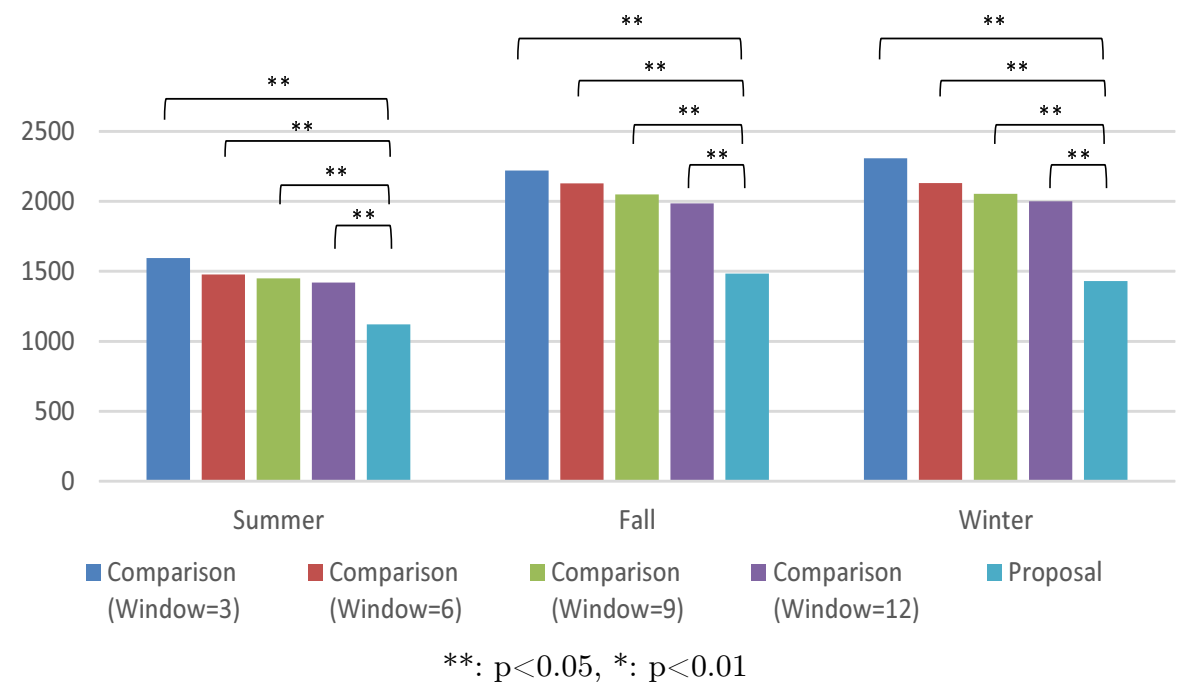

Fig. 11. Results for the MSE and its paired t-test in estimating the number $\times$ dwell time of visitors by each method for each season

Fig. 12, 13, and 14 show an example of the results and length of time window selected by our proposal in estimating the number $\times$ dwell time of visitors on a certain weekday in summer, fall, and winter, respectively.

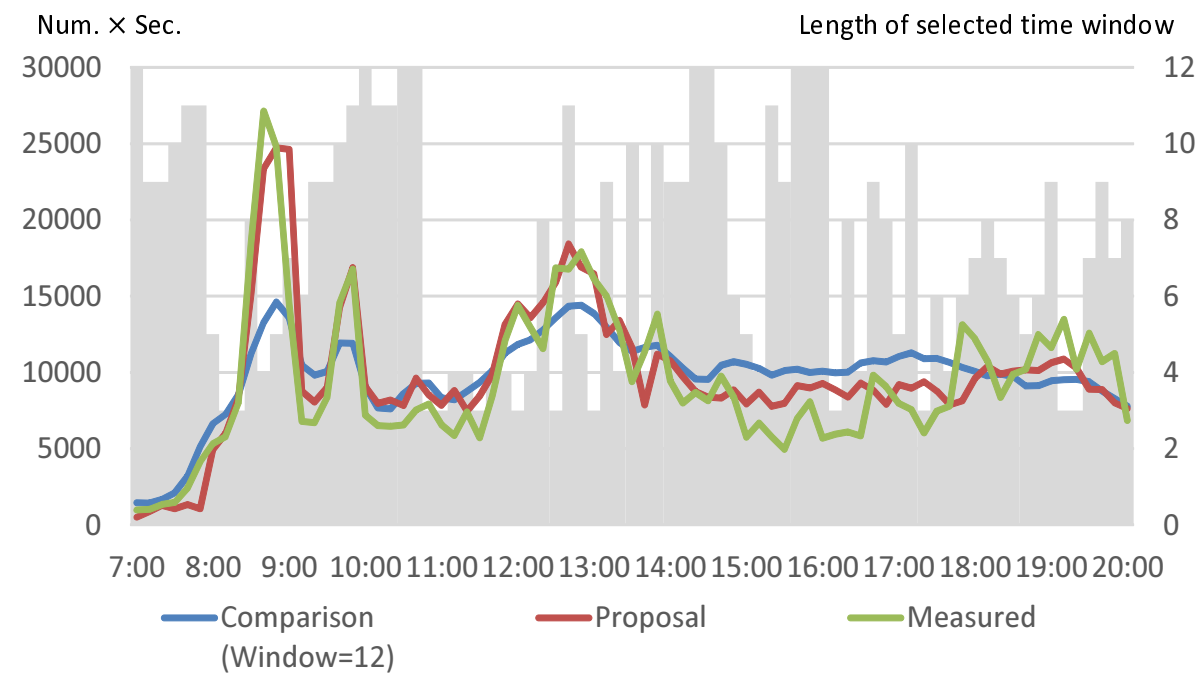

Fig. 12. Results of each method for estimating the number $\times$ dwell time of visitors and length of time window selected by our proposal on a certain weekday in summer 
Num. $\times$ Sec.

Length of selected time window

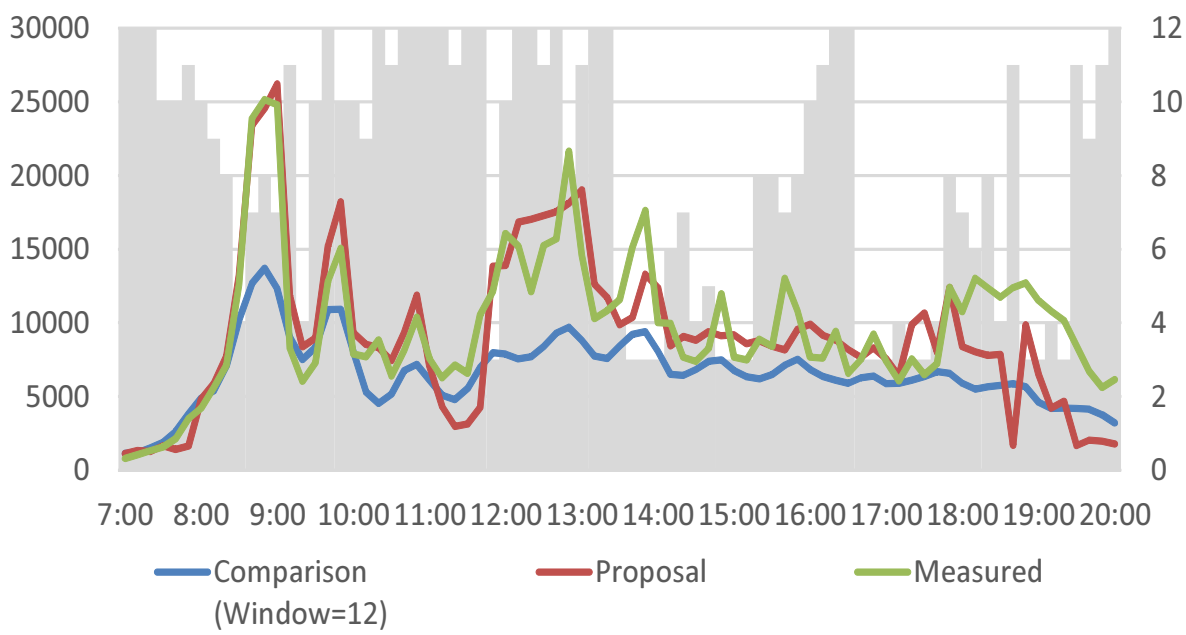

Fig. 13. Results of each method for estimating the number $\times$ dwell time of visitors and length of time window selected by our proposal on a certain weekday in fall

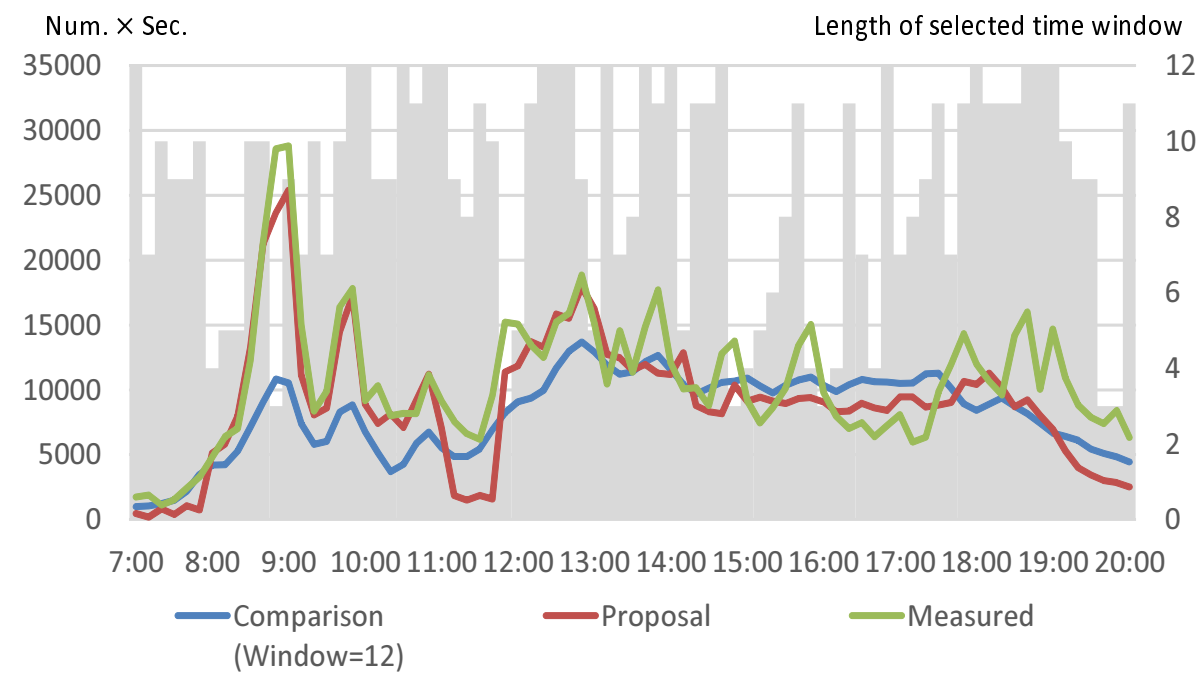

Fig. 14. Results of each method for estimating the number $\times$ dwell time of visitors and length of time window selected by our proposal on a certain weekday in winter 


\subsection{Discussion}

The results clarify that our proposal accurately estimates the number of visitors, especially rapid increases and decreases of visitors at around 9:00 and 12:00 by selecting the length of the time window in each time and season. Especially, it seems that our proposal tends to select a shorter time window when the number of visitors changes drastically, i.e. from 8:00 to 10:00 or 18:00 to 20:00. This seems to be because our proposal can adapt to differences in the speed of change and changes in the differences between the CO2 concentration and the number of visitors by automatically deciding the best length of the time window in the model.

Fig, 4 and 10 show that not only our proposal but also comparison methods estimate the number and number $\times$ dwell time of visitors in summer more accurately than in fall and winter. This seems to be because of the difference of HVAC operation in each season. Specifically, HVAC supplies cool air almost all the time in summer, but it supplies either cool or warm air to adjust the indoor temperature affected by the outdoor temperature, which might change more in fall or winter than in summer. Our proposal is effective in these three seasons as shown in Fig. 3, 4, 5, 9, 10, and 11. However, to improve the accuracy, parameters related to supply air temperature should be added to our proposal.

Here we also discuss use-case of outputs from our proposal. Our proposal can estimate both the number of visitors and number $\times$ dwell time of visitors. They generally have a positive correlation, but the difference in their changes appeared in the target lobby as shown in Figs. 8 and 14 that are measured on the same winter weekday. If we focus on both data, they have different peaks. For example, the number of visitors peaks at about 10:00 whereas the number $\times$ dwell time of visitors peaks at about 9:00. This suggests that the average dwell time is longer at 9:00 than that at 10:00. It also suggests that managers and operators of the facility can understand how to best use the lobby at any time in a day. In this example, the lobby might be crowded with many visitors who might work in offices in the facility, so someone might need to guide visitors to walk in lines at 9:00 on weekdays, or facility manager might control heating function in HVAC system to be comfortable environment for waiting people at 9:00 and walking people at 10:00 respectively based on 20]. Therefore, our proposal should also help facility managers and operators to work more efficiently and effectively without installing, operating, or maintaining additional sensors and systems.

Lastly, we describe the important limitation of our contributions. For the target building in this paper, most visitors are adults, who emit more CO2 than children . Therefore, if more children visit the target building, our proposal will need its parameters to be adjusted, other features will need to be added such as school schedules, or the algorithm will need to be improved.

\section{Conclusion}

In this paper, we tried to estimate the number and dwell time of visitors in a large room or space with CO2 concentration measured by building energy management

system (BEMS) or building automation system (BAS) and without additional sensors or systems. Our contributions are as follows. 
- We proposed partial modeling with a variable time window, which can select the most suitable length of a time window in the model to overcome the difference in the speed of changes between the CO2 concentration and the number and dwell time of people and changes in the difference caused by a change in the amount of ventilation. We used our proposal to estimate both the number of visitors and number $\times$ dwell time of visitors in a large-scale indoor space with more than 500 people in summer, fall, and winter.

- We demonstrated the effectiveness of our proposal in summer, fall, and winter and investigated the differences in its effectiveness in those three different seasons. We also clarified that our proposed method satisfies all four requirements: 1) estimating the number and dwell time of visitors in a large room or space, 2) using only existing sensors in a facility, 3) estimating large numbers of people (more than 500) who go in/out of a large space or room, and 4) estimating the number and dwell time of visitors in any season.

- We also demonstrated that outputs of our proposal are useful and effective for facility managers and operators to understand how to best use a facility at any time every day without needing to incur additional installation and maintenance costs with the example of heating-ventilation-air conditioning (HVAC) operation in winter.

Future work includes evaluating how well our proposal estimates the number and dwell time of visitors including more children and estimate them in a large room and space with multiple ventilation systems or in divided areas in a large room or space.

\section{References}

1. Araszkiewicz, K., Digital Technologies in Facility Management - The state of Practice and Research Challenges, Procedia Engineering, 196, pp. 1034-1042 (2017).

2. Goyal, S., Barooah, P., and Middelkoop, T., Experimental study of occupancybased control of HVAC zones. Applied Energy, 140, pp.75-84 (2015).

3. Ehrlich, C. R. and Blankenbach, J., Pedestrian localisation inside buildings based on multi-sensor smartphones. Proc. IEEE UPINLBS 2018, pp. 1-10 (2018).

4. Teixeira, T and Savvides, A., Lightweight people counting and localizing for easily deployable indoors WSNs. IEEE Journal of Selected Topics in Signal Processing, 2(4), pp.493-502 (2008).

5. Panangadan, A., Matarić, M. and Sukhatme, G. S., Tracking and Modeling of Human Activity Using Laser Rangefinders, International Journal of Social Robotics, 2, pp.95-107 (2010).

6. Arief-Ang, I. B., Hamilton, M., and Salim, F. D., A scalable room occupancy prediction with transferable time series decomposition of $\mathrm{CO} 2$ sensor data, $A C M$ Trans. Sensor Networks (TOSN), 14(3-4), pp.1-28 (2018).

7. Kastner, W., Neugschwandtner, G., Soucek, S. and Newman, H. M., Communication systems for building automation and control. Proc. IEEE, 93(6), pp.1178-1203 (2005).

8. Manic, M., Wijayasekara, D., Amarasinghe, K., and Rodriguez-Andina, J. J., Building energy management systems: The age of intelligent and adaptive buildings, IEEE Industrial Electronics Magazine, 10(1), pp.25-39 (2016). 
9. Shackleton, J., VanVoorst, B., and Hesch, J., Tracking people with a 360-degree lidar, Proc. 7th IEEE AVSS, pp. 420-426 (2010).

10. Longo, E., Redondi, A. E. and Cesana, M., Accurate occupancy estimation with WiFi and bluetooth BLE packet capture, Computer Networks, 163(9), 106876 (2019).

11. Mateos-Sánchez, M., Berjón-Gallinas, R., Beato-Gutiérrez, M. E., and FermosoGarcía, A. M., A Tool to Calculate the Level of Occupancy in Indoor and Outdoor Spaces Using BLE and Open Data to Be Published in Real-Time. Sensors, 20(14), 3916 (2020).

12. Zou, H., Zhou, Y., Yang, J. and Spanos, C. J., Device-free occupancy detection and crowd counting in smart buildings with WiFi-enabled IoT, Energy and Buildings, 174(1), pp.309-322 (2018).

13. Ebadat, A., Bottegal, G., Varagnolo, D., Wahlberg, B., and Johansson, K. H., Estimation of building occupancy levels through environmental signals deconvolution.,Proc. the 5th ACM Workshop on Embedded Systems For Energy-Efficient Buildings, pp.1-8 (2013).

14. Alam, A. G., Rahman, H., Kim, J. K., and Han H., Uncertainties in neural network model based on carbon dioxide concentration for occupancy estimation, $J$. Mechanical Science and Technology, 31, pp.2573-2580 (2017)

15. Tsunoda, K., Arai, N., and Obana, K., Estimating Number and Dwell Time of Visitors from CO2 Concentration using Partial Modeling with Variable Time Window, Proc. IEEE ai4i 2020, pp. 14-18 (2020).

16. Atkeson, C. G., Moore, A. W., and Schaal, S., Locally weighted learning. In Lazy learning, pp. 11-73. Springer, Dordrecht (1997).

17. Bontempi, G., Birattari, M. and Bersini, H., Lazy learning for local modelling and control design, International Journal of Control, 72(7-8), pp.643-658 (1999).

18. The Japan Meteorological Agency, Overview of Japan's climate, https://www . data.jma.go.jp/gmd/cpd/longfcst/en/tourist_japan.html.

19. Drucker, H., Burges, C. J., Kaufman, L., Smola, A. and Vapnik, V.,, Support vector regression machines, Advances in neural information processing systems, 9 , pp. 155-161 (1996).

20. The International Organization for Standardization, ISO 7730:2005 Ergonomics of the thermal environment - Analytical determination and interpretation of thermal comfort using calculation of the PMV and PPD indices and local thermal comfort criteria, https://www .iso.org/obp/ui/\#iso:std:iso:7730:ed-3:v1:en. 